\title{
Performance of Optical Heterodyne PSK Systems with Costas Loop in Multichannel Environment for Nonlinear Second-Order PLL Model
}

Ivan B. Djordjevic and Mihajlo C. Stefanovic, Associate Member, IEEE

\begin{abstract}
Using the nonlinear second-order phase-locked loop (PLL) model the performance of the heterodyne coherent optical phase shift keying (PSK) systems with Costas loop in multichannel environment is considered in this paper for the first time. The shot noise of the corresponding photodiodes and adjacent channel interferences are described through the signal-to-noise ratio (SNR) in the loop bandwidth, while the laser phase noise is described through the normalized frequency fluctuations instead of the phase ones. The theory and results presented in this paper can be applied when analyzing and optimizing the performance in the region where the linear PLL model is not enough good.
\end{abstract}

Index Terms - Coherent optical systems, Costas loop, error probability, multichannel environment, nonlinear phase-locked loop (PLL) model.

\section{INTRODUCTION}

$\mathbf{T}$ HE use of coherent optical communication systems offers the promise of significant improvements in receiver performance compared with intensity modulation/direct detection ones [1]. The cost of these improvements is in the complexity increasing. Since phase shift keying (PSK) heterodyne detection with synchronous demodulation achieves the most sensitive detection among heterodyne schemes, it can be utilized for frequency division multiplexing (FDM) systems. However, in a multichannel environment, coherent optical receivers suffer a performance degradation [2]-[4] coming from several phenomena, including direct detection, signalcross-signal and adjacent channel interferences. It has been shown [2] that balanced reception eliminate the first two kinds of interference and also cancel excess intensity noise present in the local oscillator [3].

Successful information transmission through a phase coherent systems requires, by definition, a receiver capable of determining or estimating the phase and frequency of the received signal with as little error as possible. In the coherent communications, synchronization and tracking are generally accomplished by a crosscorrelating a locally generated reference signal with the received signal to produce the error measurement. In practice, quite often the phaselocked loop (PLL) is used in providing the desired reference signal [1]-[3], [5]-[10]. Frequently, PLL must operate in such conditions where external fluctuations due to additive noise

Manuscript received January 5, 1999; revised July 28, 1999.

The authors are with Faculty of Electronic Engineering, University of Nis, Nis 18000 Yugoslavia.

Publisher Item Identifier S 0733-8724(99)09670-X. are so intense that the classical linear PLL theory neither adequately characterize loop performance nor explain loop behavior. The direct linearization cannot be used in loop performance explanation and characterization in the region of the operation in many practical solutions. So, the analytical approach in developing an exact nonlinear PLL theory, based on Fokker-Planck theory was investigated in [7]-[9] and this nonlinear model is used in this paper. Even when the signal at the PLL input is completely known, there is some uncertainty created by additive noise that accompanies the useful signal at the input. For the nonlinear PLL model, this uncertainty in the literature is described by the probability density function of the phase error.

Taking the laser phase noise, the shot noise of the corresponding photodiodes and the adjacent channel interferences into account the performance of the coherent optical PSK systems with Costas loop in multichannel environment was considered in [3]. The analysis, presented in that paper, was given for the linear second-order PLL model.

The performance of the coherent optical systems with a PLL in a multichannel environment for the nonlinear PLL model was considered in [5]. However, due to complexity of the problem, the analysis was given only for the firstorder PLL, i.e., omitting the loop filter in the PLL subsystem. Unfortunately, the phase-locked loop stability is very hard to achieve with the first-order loop. Strictly speaking, the first-order loop does not really exist [11].

In this paper the performance analysis of the coherent optical systems with Costas loop as constituent part of the receiver in the multichannel environment for the nonlinear secondorder PLL model is considered for the first time. The shot noise of the corresponding photodiodes and adjacent channel interferences are taken into consideration through the signalto-noise ratio (SNR) in the loop bandwidth, whereas the laser phase noise is described through the carrier frequency offset instead of the phase fluctuations. The connection among the global system characteristics (for example, the system bit-error rate) and needed system elements (the loop filter elements, the laser linewidth, etc.) is established. In determining the receiver error probability the method of conditional probabilities is used-that is, we develop expressions for the particular parameter, distribution or error probability, conditioned on the phase error, being fixed over a bit duration $T$. By averaging over this condition, which is random, we determine the behavior of interest. 


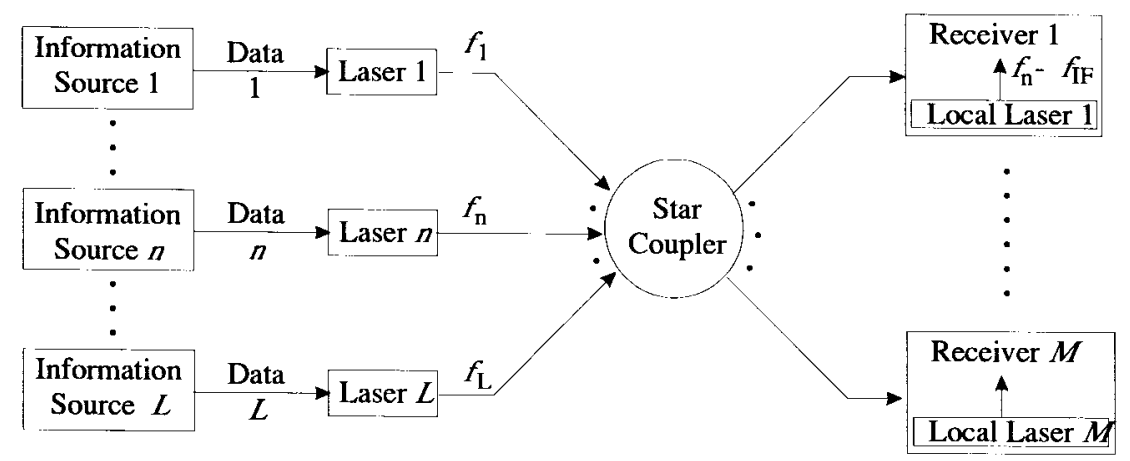

(a)

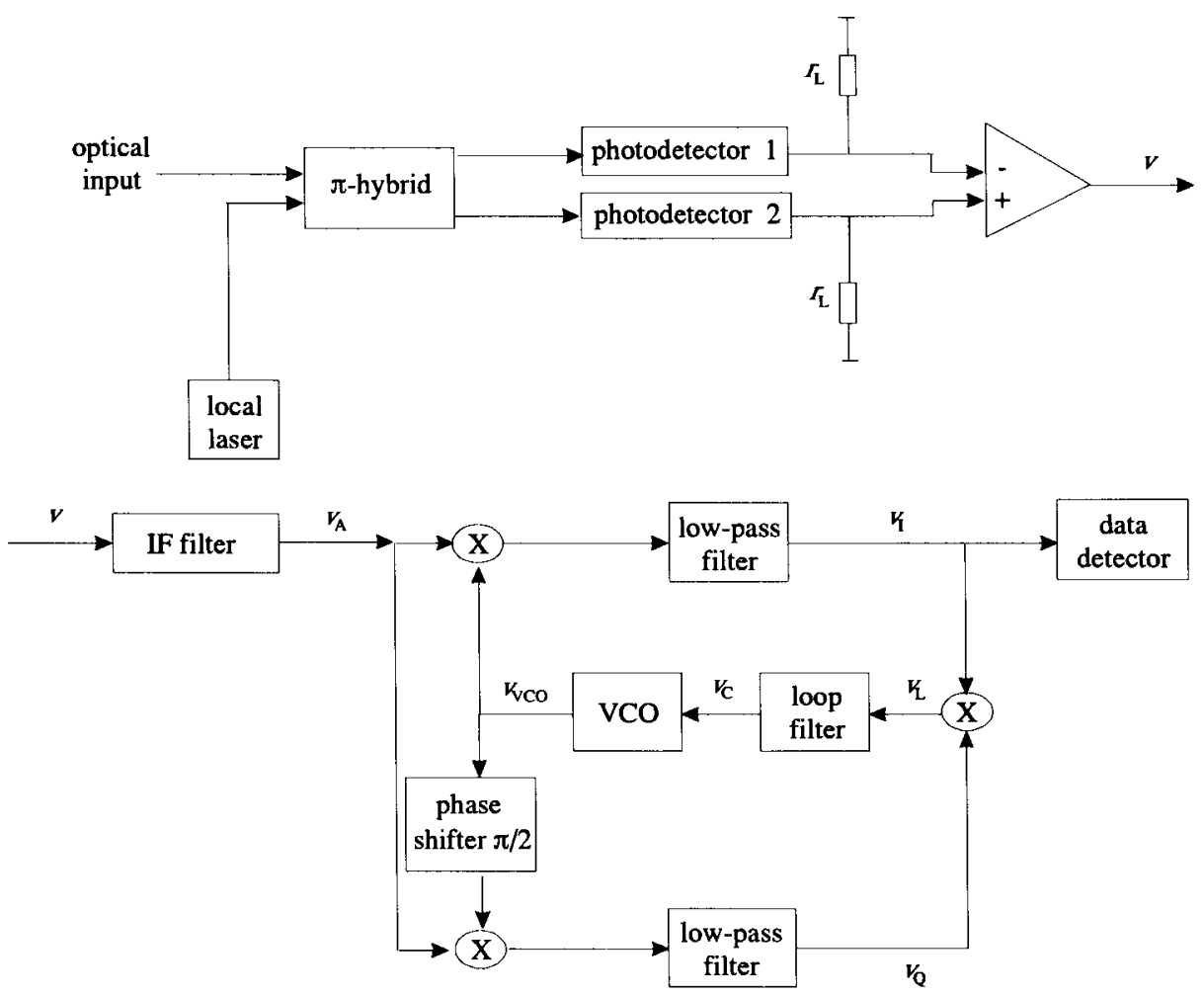

(b)

Fig. 1. (a) Multichannel coherent optical heterodyne PSK system model. (b) Model of heterodyne receiver with Costas loop.

The rest of the paper is organized as follows. Section II contains the theoretical basis for the performance evaluating of the heterodyne coherent optical PSK system with Costas loop receiver when the nonlinear second-order PLL model is taken into consideration. Section III contains numerical results analysis and discussion. Finally, Section IV offers some conclusions.

\section{System Model and Performance Determination}

The block diagram of the multichannel frequency division multiplexing (FDM) system under investigation is shown in Fig. 1(a), while the corresponding receiver model of Fig. 1(a) is the heterodyne receiver with Costas loop as shown in Fig. 1(b). The purpose of the presented system is to make all $L$ information sources available to $M$ receivers that can be achieved by using $L$ lasers tuned to different frequencies with separation $D$ between two adjacent optical channels. The frequency of the $n$th optical channel is given by

$$
f_{n}=f_{1}+(n-1) D, \quad 1 \leq n \leq L .
$$

The signals of the $L$ lasers are combined by using a $L \times M$ star coupler and sent to $M$ receivers. Each receiver can be tuned to the $n$th channel by adjusting the frequency of the local oscillator (LO) to $f_{L O}=f_{n}-f_{I F}$, with $f_{\mathrm{IF}}$ being the intermediate frequency (IF). The intermediate frequency should be kept smaller than $D$ such that $f_{\mathrm{LO}}$ lies between the carrier frequency of the desired channel and that of the adjacent channel [2]-[3].

The model of heterodyne receiver with Costas loop is shown in Fig. 1(b). In the analysis that follows the following assumptions are made: 1) the interchannel interference is negligibly small when the intermediate frequency is very small compared to the channel spacing in optical domain, 2) the crosstalk interference to the $n$th channel is mainly generated 
from the $(n-2)$ th, $(n-1)$ th, $(n+1)$ th and $(n+2)$ th channels, and 3 ) the intermediate filter passes the signal of carrier frequency undistorted. These assumptions are reasonable for most of the practical systems [4].

Under the assumption that all signals in the multichannel environment have the same polarization state corresponding to the local laser polarization state, the incoming optical signal $S$ and local laser outcoming signal $L O$ can be presented as

$$
\begin{aligned}
S= & \sqrt{2 P_{S}} \cos \left[\omega_{n} t+\Delta_{n}(t)+\vartheta_{n}(t)\right] \\
& +\sum_{k=1, k \neq n}^{L} \sqrt{2 P_{S k}} \cos \left[\omega_{k} t+\Delta_{k}(t)+\vartheta_{k}(t)\right], \\
L O= & \sqrt{2 P_{L O}} \cos \left[\omega_{L O} t+\vartheta_{L O}(t)\right]
\end{aligned}
$$

where $L$ is the total number of the optical channels, $P_{\mathrm{Sk}}=$ $P_{\mathrm{S}}(k=1,2, \cdots, n-1, n+1, \cdots, L)$ and $P_{\mathrm{LO}}$ are the powers of the received signal and local oscillator outcoming signal, respectively; $\Delta_{\mathrm{k}}(t)$ is the angle modulation of the $k$ th signal. Finally, $\vartheta_{\mathrm{k}}(t)$ and $\vartheta_{\mathrm{LO}}(t)$ are the laser phase noises of the $k$ th signal and local oscillator, respectively, caused by the finite laser linewidth. To combine the signal and local oscillator fields, optical hybrid is used. The optical hybrid output signals are detected by two photodiodes. The intermediate filter (IF) input signal can be written as

$$
\begin{aligned}
v(t)= & 2 r_{L} R \sqrt{P_{S} P_{L O}} d_{n}(t) \cos \left[\omega_{I F} t+\Psi(t)\right] \\
& +n(t)+2 r_{L} R 2 \sum_{k=1, k \neq n}^{L} \sqrt{P_{S} P_{L O}} d_{k}(t) \\
& \cdot \cos \left(\omega_{I F} t+2 \pi(k-n) D t+\Psi_{k, \mathrm{LO}}(t)\right)
\end{aligned}
$$

where $R$ is the detector responsivity, $\Psi(t)$ combines the observed signal (the $n$th channel signal, the first term in (1)) and local oscillator phase noises, and $\omega_{I F}=2 \pi f_{I F}$ is the difference between the $n$th channel optical signal frequency $\left(\omega_{n}\right)$ and local laser output signal one $\left(\omega_{L O}\right)$. With $r_{L}$ is denoted the load resistance. $\Psi_{\mathrm{k}, \mathrm{LO}}(t)$ combines the $k$ th channel signal phase noise and local oscillator one. Since $\Delta_{\mathrm{k}}(t)$ is either 0 or $\pi$ for the bit period, the angle modulation state can be presented by $d_{\mathrm{k}}(t)$, which is +1 or -1 , depending on the transmitted bit. The third term of (2) should be filtered by the IF filter. $n(t)$ is the shot noise process with double sided power spectral density (PSD)

$$
\operatorname{PSD}_{n}(f)=q r_{L}^{2} R\left(P_{L O}+L P_{S}\right)
$$

with $q$ being an electron charge. It was shown that the power penalty, caused by the excess shot noise with PSD $q r_{L}^{2} R(L-1) P_{S}$, is less than $1 \mathrm{~dB}$ for $L<200$ channels and moderate LO power values $\left(P_{L O} / P_{S} \cong 30 \mathrm{~dB}\right)$ [2]-[3]. Hence, the approximation $\operatorname{PSD}_{n}(f) \cong q r_{L}^{2} R P_{L O}$ is assumed to be valid. The differential amplifier output signal is filtered by a bandpass filter with bandwidth $2 B=2 R_{\mathrm{b}}$ ( $R_{\mathrm{b}}$-the bit rate), corresponding to the main lobe in the PSK signal. The filter passes the useful signal undistorted. The IF filter output signal has the form

$$
\begin{aligned}
v_{A}(t)= & 2 r_{L} R \sqrt{P_{S} P_{L O}} d_{n}(t) \cos \left[2 \pi f_{I F} t+\Psi(t)\right] \\
& +s(t)+n_{I F}(t)
\end{aligned}
$$

where $s(t)$ represents the filtered adjacent channels and it is modeled by using the Gaussian approximation

$$
s(t)=a(t) \cos \omega_{I F} t-b(t) \sin \omega_{I F} t
$$

where

$$
\begin{aligned}
E[a(t)]= & E[b(t)]=0, \quad R_{a}(\tau)=R_{b}(\tau), \\
R_{a b}(\tau)= & -R_{b a}(\tau), \quad R_{a b}(0)=0, \\
\operatorname{PSD}_{a}(f)= & \operatorname{PSD}_{b}(f)=\frac{1}{2} \sum_{j=1, j \neq n}^{K}\left[T \operatorname{sinc}^{2}\left(f-D_{j}\right)\right. \\
& \left.+T \operatorname{sinc}^{2}\left(f-D_{j}\right)\right]
\end{aligned}
$$

where $D_{j}(j=1,2, \cdots, K-1)$ are the electrical spacings. The notation used here is $R_{x}(\tau)=E[x(t) x(t+\tau)], R_{x y}(\tau)=$ $E[x(t) y(t+\tau)],(E[]$ - the mathematical expectation operator), $\operatorname{PSD}_{x}[f]=\operatorname{PSD}\left[R_{x}(\tau)\right]$ (PSD [] - the power spectral density operator). The function $\operatorname{sinc}(x)$ is defined as

$$
\operatorname{sinc}(x)=\sin (\pi T x) /(\pi T x) .
$$

This approximation, which validity was considered in other papers [2]-[3], leads to simple expression for the error probability. $d_{n}(t)$ is +1 or -1 depending on the transmitted bit and $n_{\mathrm{IF}}(t)$ is the IF filter filtered version of the photodiode shot noise $n(t)$

$$
n_{I F}(t)=N_{c}(t) \cos \omega_{I F} t-N_{s}(t) \sin \omega_{I F} t
$$

with $N_{c}(t)$ and $N_{s}(t)$ being the in-phase and quadrature components of the narrowband process having the properties

$$
\begin{aligned}
E\left[N_{c}(t)\right] & =E\left[N_{s}(t)\right]=0 \\
R_{N_{c}}(\tau) & =R_{N_{c}}(\tau)=R_{N_{c} N_{s}}(\tau)=0 \\
\operatorname{PSD}_{N_{c}}(f) & =\operatorname{PSD}_{N_{s}}(f)=2 q r_{L}^{2} R P_{L O}, \\
& \text { for }|f|<B .
\end{aligned}
$$

The voltage controlled oscillator (VCO) output signal can be written as

$$
v_{\mathrm{VCO}}(t)=A_{\mathrm{VCO}} \cos \left[2 \pi f_{I F} t+\phi_{\mathrm{VCO}}(t)\right]
$$

where $A_{\mathrm{VCO}}$ and $\phi_{\mathrm{VCO}}$ are the $\mathrm{VCO}$ amplitude and phase, respectively. The $\mathrm{VCO}$ phase is given by

$$
\phi_{\mathrm{VCO}}(t)=G_{\mathrm{VCO}} \int_{-\infty}^{t} v_{c}\left(t^{\prime}\right) d t^{\prime}
$$

where $G_{\mathrm{VCO}}$ is the $\mathrm{VCO}$ gain and $v_{c}(t)$ is the VCO input signal

$$
v_{c}(t)=V_{L}(t) * f(t)
$$

with $v_{L}(t)$ being the multiplier output signal and $f(t)$ being the loop filter impulse response. With $*$ is denoted the convolution operation.

The corresponding the low-pass filters output signals are

$$
\begin{aligned}
v_{I}(t)= & A_{\mathrm{VCO}} r_{L} R \sqrt{P_{S} P_{L O}} d_{n}(t) \cos [\varphi(t)]+n_{I}(t)+c_{I}(t) \\
v_{Q}(t)= & A_{\mathrm{VCO}} r_{L} R \sqrt{P_{S} P_{L O}} d_{n}(t) \\
& \cdot \sin [\varphi(t)]+n_{Q}(t)+c_{Q}(t)
\end{aligned}
$$


where $\varphi(t)=\Psi(t)-\phi_{\mathrm{VCO}}$ is the total phase error, whereas

$$
\begin{aligned}
n_{I}(t) & =n_{I F}(t) v_{\mathrm{VCO}}(t), \quad c_{I}=c(t) v_{\mathrm{VCO}}(t) \\
n_{Q}(t) & =n_{I F}(t) v_{\mathrm{VCO}}(t) e^{j \pi / 2} \\
c_{Q}(t) & =, c(t) v_{\mathrm{VCO}}(t) e^{j \pi / 2} .
\end{aligned}
$$

The subscripts $I$ and $Q$ refer to the in-phase and quadrature components of the narrowband noise $n_{I F}(t)$ i.e. crosstalk $c(t)$. Under assumption that the low-pass filters have the same rectangular frequency response with double sided bandwidth $2 B=2 R_{\mathrm{b}}$. Therefore, the multiplier output signal can be represented as

$$
v_{L}(t)=v_{I}(t) v_{Q}(t)=\frac{G_{T}^{2}}{2} \sin [2 \varphi(t)]+N(t)
$$

where

$$
\begin{aligned}
N(t)= & S_{I} n_{Q}+S_{I} c_{Q}+n_{I} S_{Q}+n_{I} n_{Q}+n_{I} c_{Q} \\
& +c_{I} S_{Q}+c_{I} n_{Q}+c_{I} c_{Q}
\end{aligned}
$$

the equivalent noise, $G_{T}=A_{\mathrm{VCO}} r_{L} R \sqrt{P_{S} P_{L O}}, S_{I}=$ $G_{T} d_{n}(t) \cos \varphi(t)$, and $S_{Q}=G_{T} d_{n}(t) \sin \varphi(t)$.

Starting form (7), (8) and (11) we may describe the PLL subsystem operation by the stochastic differential equation

$$
\begin{aligned}
\frac{d \phi_{\mathrm{VCO}}(t)}{d t} & =G_{\mathrm{VCO}} \cdot v_{c}(t)=G_{\mathrm{VCO}} \cdot v_{L}(t) * f(t) \\
& =\frac{1}{2} G_{\mathrm{VCO}} G_{T}^{2} \cdot\{\sin [2 \varphi(t)]+N(t)\} * f(t) \\
& =\frac{1}{2} G_{\mathrm{VCO}} G_{T}^{2} \cdot F(p)\{\sin [2 \varphi(t)]+N(t)\}
\end{aligned}
$$

where $F(p)$ is the loop filter transfer function, with $p=(d / d t)$ being the Heaviside operator. This the stochastic differential equation is equivalent to the differential equation (A1) defined in Appendix A.

According to the results presented in [6]-[10] and Appendix A the conditional steady-state probability density function (pdf) of the modulo $2 \pi$ reduced phase error $\Phi(\Phi=2 \varphi, \varphi$-the actual phase error being tracked by the loop) for the nonlinear second-order PLL model can be written as

$$
\begin{aligned}
p\left(\Phi \mid \Omega_{n}\right)= & \frac{\exp \left[\alpha \cos \Phi+\beta\left(\Omega_{n}\right) \Phi\right]}{4 \pi^{2} \exp \left[-\pi \beta\left(\Omega_{n}\right)\right]\left|I_{j \beta\left(\Omega_{n}\right)}(\alpha)\right|^{2}} \\
& \cdot \int_{\Phi}^{\Phi+2 \pi} \exp \left[-\alpha \cos x-\beta\left(\Omega_{n}\right) x\right] d x
\end{aligned}
$$

where $I_{v}(x)$ is modified Bessel function of the first kind of the order $v$ and the argument $x$. (In writing conditional pdf's, conditional variables are written to the right of the vertical bar). The region of definition for $\Phi$ is any interval of width $2 \pi$ about any lock point $2 n \pi$, with $n$ any integer. The parameters $\alpha$ and $\beta\left(\Omega_{n}\right)$ that characterize (13), when the loop filter is described by the transfer function $F(s)=\left(1+s \tau_{2}\right) /\left(1+s \tau_{1}\right)$, are related to the system parameters through

$$
\begin{aligned}
\alpha & =\frac{r+1}{r} \rho^{\prime}-\frac{1-F}{r \sigma_{G}^{2}}, \quad F=\tau_{2} / \tau_{1}, \quad r=A^{2} K F \tau_{2} \\
\beta\left(\Omega_{n}\right) & =\frac{r+1}{r} \frac{\rho^{\prime}}{F}\left(2 \Omega_{n}-\overline{\sin \Phi}\right)+\alpha \overline{\sin \Phi}
\end{aligned}
$$

where $\rho^{\prime}=(\rho / 4) S_{L}$ is the effective SNR in the loop bandwidth, with $S_{\mathrm{L}}$ being the squaring loss that can be found, starting from [9] (in the case of the ideal low-pass filters), as

$$
S_{L}=\frac{1}{1+\frac{R_{b}}{\rho B_{\text {PLL }}}} .
$$

With $B_{\text {PLL }}$ is denoted the equivalent loop noise bandwidth

$$
B_{\text {PLL }}=\frac{1}{2} \int_{-\infty}^{\infty}\left|H_{\text {PLL }}(f)\right|^{2} d f
$$

with $H_{\text {PLL }}(f)$ being the closed loop transfer function

$$
H_{\mathrm{PLL}}(s)=\frac{G_{T}^{2} G_{\mathrm{VCO}} F(s)}{s+G_{T}^{2} G_{\mathrm{VCO}} F(s)} .
$$

For the loop filter with transfer function $F(s)=\left(1+s \tau_{2} / 1+\right.$ $\left.s \tau_{1}\right)$, under assumption that the loop gain $G_{T}^{2} G_{\mathrm{VCO}} \gg 1$ then $\left|H_{\text {PLL }}(f)\right|^{2}$ is given by

$$
\left|H_{\text {PLL }}(f)\right|^{2}=\frac{1+4 \zeta^{2} f^{2} / f_{\text {nat }}^{2}}{\left(1-\frac{f^{2}}{f_{\text {nat }}^{2}}\right)+4 \zeta^{2} \frac{f^{2}}{f_{\text {nat }}^{2}}}
$$

where $f_{\text {nat }}$ and $\zeta$ are the natural frequency and the damping factor, respectively, defined as

$$
f_{\text {nat }}=\frac{1}{2 \pi} \sqrt{\frac{G_{T}^{2} G_{\mathrm{VCO}}}{\tau_{1}}}
$$

and

$$
\zeta=\pi f_{\text {nat }} \tau_{2} \text {. }
$$

Therefore, the equivalent loop noise bandwidth $B_{\text {PLL }}$ can be written in the form

$$
B_{\mathrm{PLL}}=\frac{\pi f_{\mathrm{nat}}\left(1+4 \zeta^{2}\right)}{4 \zeta} .
$$

$\rho$ is the equivalent SNR in loop bandwidth of standard secondorder PLL and using results presented in [7] can be calculated by

$$
\rho=1 / \sigma_{\varphi}^{2}
$$

where $\sigma_{\varphi}^{2}$ is the total phase error variance in the linear sense (see paper [3] for derivation)

$$
\begin{aligned}
\sigma_{\varphi}^{2}= & \frac{\pi\left(1+4 \zeta^{2}\right) \Delta \nu}{8 \zeta^{2} B_{\mathrm{PLL}}} \\
& +\left(\frac{q^{2} B}{R^{2} P_{S}^{2}}+\frac{q}{R P_{S}}+\frac{q}{4 R P_{S}} N_{3}+\frac{N_{4}}{4}+\frac{N_{\tilde{3}}}{16}\right) B_{\text {PLL }}
\end{aligned}
$$

where $N_{3}, N_{4}$, and $N_{5}$ are defined, respectively, (see the paper [3] for derivation) as

$$
\begin{aligned}
N_{3}= & \int_{-B}^{B} \int_{j=1, j \neq n}^{L}\left[T \operatorname{sinc}^{2}\left(f-D_{j}\right)\right. \\
& \left.+T \operatorname{sinc}^{2}\left(f+D_{j}\right)\right\rfloor d f \\
N_{4}= & \int_{-B}^{B} \int_{j=1, j \neq n}^{L}\left[T \operatorname{sinc}^{2}\left(f-D_{j}\right)+T \operatorname{sinc}^{2}\left(f+D_{j}\right)\right] \\
& \cdot T \operatorname{sinc}^{2} f d f
\end{aligned}
$$


and

$$
\begin{aligned}
N_{5}= & 2 \int_{-B}^{B}\left\{\sum_{j=1, j \neq n}^{L}\left[T \operatorname{sinc}^{2}\left(f-D_{j}\right)\right]\right. \\
& \left.\cdot \sum_{j=1, j \neq n}^{L}\left[T \operatorname{sinc}^{2}\left(f+D_{j}\right)\right]\right\} d f .
\end{aligned}
$$

The electrical spacings are given by [3] $D_{1}=D-2 R_{b}$, $D_{2}=D, D_{3}=2 D-2 R_{b}$ and $D_{4}=2 D$, with $D$ being the optical frequency separation between two adjacent channels. The contributions of other channels are negligible. Similarly as in [7]-[9], in determining the SNR in loop bandwidth the linearized PLL model was used. Otherwise, the loop bandwidth can not be defined in the nonlinear sense (see the corresponding differential equation in Appendix A). With $\Omega_{n}=\Omega / A^{2} K\left(A^{2} K\right.$-the normalized loop gain, $A^{2} K=$ $\left.\frac{1}{2} G_{\mathrm{VCO}} G_{T}^{2}\right)$ is denoted the normalized frequency offset, where $\Omega=\omega_{I F}-\omega_{\mathrm{VCO}}\left(\omega_{I F}\right.$-the Costas loop incoming signal frequency, $\omega_{\mathrm{VCO}}-$ the voltage controlled oscillator output signal frequency). When the PLL is in lock-in-range the following is valid

$$
\omega_{I F}=\omega_{\mathrm{VCO}}+\dot{\varphi}
$$

where $\varphi(t)$ represents the actual phase error being tracked by the loop $(\dot{\varphi}=d \varphi(t) / d t)$. Therefore, the normalized frequency offset can be written in the form $\Omega_{n}=\dot{\varphi} / A^{2} K$. Knowing that $\dot{\varphi}$ has a zero-mean Gaussian distribution [1], [10] it is no difficult to show that $\Omega_{n}$ has also a zero-mean Gaussian distribution with the variance

$$
\sigma_{\Omega_{n}}^{2}=4 \pi \Delta \nu /\left(A^{2} K\right)^{2}
$$

under assumption that the corresponding laser linewidths of the transmitter laser $\left(\Delta \nu_{T}\right)$ and the local laser $\left(\Delta \nu_{L O}\right)$ are the same $\Delta \nu_{T}=\Delta \nu_{L O}=\Delta \nu$. With $\sigma_{G}^{2}$ is denoted the variance of the stochastic process $G=\sin \Phi-\overline{\sin \Phi}$ i.e.

$$
\sigma_{G}^{2}={\overline{\sin ^{2} \Phi}}_{-\overline{\sin }^{2}}^{2}
$$

wherein the corresponding circular moments are [9]

$$
\overline{\sin \Phi}=\int_{-\pi}^{\pi} \sin \Phi \cdot p(\Phi) d \Phi=\operatorname{Im}\left[\frac{I_{1-j \beta\left(\Omega_{n}\right)}(\alpha)}{I_{-j \beta\left(\Omega_{n}\right)}(\alpha)}\right]
$$

and

$$
\begin{aligned}
\overline{\sin ^{2} \Phi} & =\int_{-\pi}^{\pi} \sin ^{2} \Phi \cdot p(\Phi) d \Phi \\
& =\frac{1-\operatorname{Re}\left[\frac{I_{1-j \beta\left(\Omega_{n}\right)}(\alpha)}{I_{-j \beta\left(\Omega_{n}\right)}(\alpha)}\right]}{2} .
\end{aligned}
$$

In order to compare the results obtained using the nonlinear PLL model to those obtained using the linear one, the same value of $B_{\text {PLL }}$ is to be used. The optimum loop bandwidth in the linear sense $B_{\text {PLL,opt }}$, opt was used in the paper [3] when determining the receiver error probability

$$
B_{\text {PLL }, \text { opt }}=\sqrt{\frac{\pi(r+1) \Delta \nu R_{b} /(2 r)}{\frac{1}{n_{p}^{2}}+\frac{1}{n_{p}}+\frac{1}{4 n_{p}} N_{3}+\frac{N_{4} R_{b}}{4}+\frac{N_{5} R_{b}}{16}}}
$$

with $r$ being the damping parameter $\left(r=4 \zeta^{2}\right), \Delta \nu$ being the laser linewidth, and $n_{p}=R P_{S} /\left(q R_{b}\right)(R$-the photodiode responsivity, $P_{\mathrm{s}}$ - the received optical power, $R_{\mathrm{b}}=1 / T$-the bit rate, $T$-the bit duration) being the average number of photons per bit. Therefore, the equivalent SNR in loop bandwidth of standard second-order PLL, using the same $B_{\text {PLL }}$ value as in the case of the linear PLL model $\left(B_{\text {PLL,opt }}\right)$, can be found by

$$
\rho=1 / \sigma_{\varphi, \min }^{2}
$$

where

$$
\begin{aligned}
\sigma_{\varphi, \text { min }}^{2}= & {\left[\frac { 2 \pi ( r + 1 ) ( \Delta \nu / R _ { b } ) } { r } \left(\frac{1}{n_{p}^{2}}+\frac{1}{n_{p}}+\frac{1}{4 n_{p}} N_{3}\right.\right.} \\
& \left.\left.+\frac{N_{4} R_{b}}{4}+\frac{N_{5} R_{b}}{16}\right)\right]^{1 / 2}
\end{aligned}
$$

is the minimum total phase error variance in the linear sense (obtained by minimizing $\sigma_{\varphi}^{2}$ per $B_{\text {PLL }}$ ).

According to [9], Appendix A and Appendix B the conditional system error probability can be obtained as

$$
\begin{aligned}
P_{e}\left(\Omega_{n}\right)= & \frac{1}{2} \int_{-\pi}^{\pi} \operatorname{erfc}[\operatorname{SNR} \cos (\Phi / 2)] \\
& \cdot \frac{\exp \left(\alpha \cos \Phi+\beta\left(\Omega_{n}\right) \Phi\right)}{4 \pi^{2} \exp \left(-\pi \beta\left(\Omega_{n}\right)\right)\left|I_{j \beta\left(\Omega_{n}\right)}(\alpha)\right|^{2}} \\
& \cdot\left[\int_{\Phi}^{\Phi+2 \pi} \exp \left(-\alpha \cos x-\beta\left(\Omega_{n}\right) x\right) d x\right] d \Phi
\end{aligned}
$$

where the SNR at the detector input SNR is defined, see Appendix B, as

$$
\mathrm{SNR}=\frac{1}{\sqrt{\frac{\mathrm{N}}{4}+\frac{1}{n_{p}}}}
$$

wherein

$$
\begin{aligned}
\mathrm{N}= & \int_{-B}^{B} \sum_{j=1, j \neq n}^{L}\left[T \operatorname{sinc}^{2}\left(f-D_{j}\right)\right. \\
& \left.+T \operatorname{sinc}^{2}\left(f+D_{j}\right)\right] \cdot \operatorname{sinc}^{2} f d f .
\end{aligned}
$$

The average error probability can be obtained by averaging the conditional error probability $P_{e}\left(\Omega_{n}\right)$ per normalized frequency offset

$$
P_{e}=\int_{-\infty}^{\infty} P_{e}\left(\Omega_{n}\right) \frac{1}{\sigma_{\Omega_{n}} \sqrt{2 \pi}} \exp \left(-\frac{\Omega_{n}^{2}}{2 \sigma_{\Omega_{n}}^{2}}\right) d \Omega_{n} .
$$

\section{NUMERICAL RESULTS}

In analysis that follows it is assumed that $r=2$ (i.e. $\zeta=1 / \sqrt{2}$ ) [1] and $F=0.002$ [7]-[9]. For such chosen parameters the open-loop gain $A^{2} K$, according to [7]-[10], can be set as

$$
A^{2} K=4 r B_{\text {PLL }, \text { opt }} /[F(r+1)] .
$$

In the case of the linear PLL model (when the phase error distribution, according to [7], is a zero-mean Gaussian process 


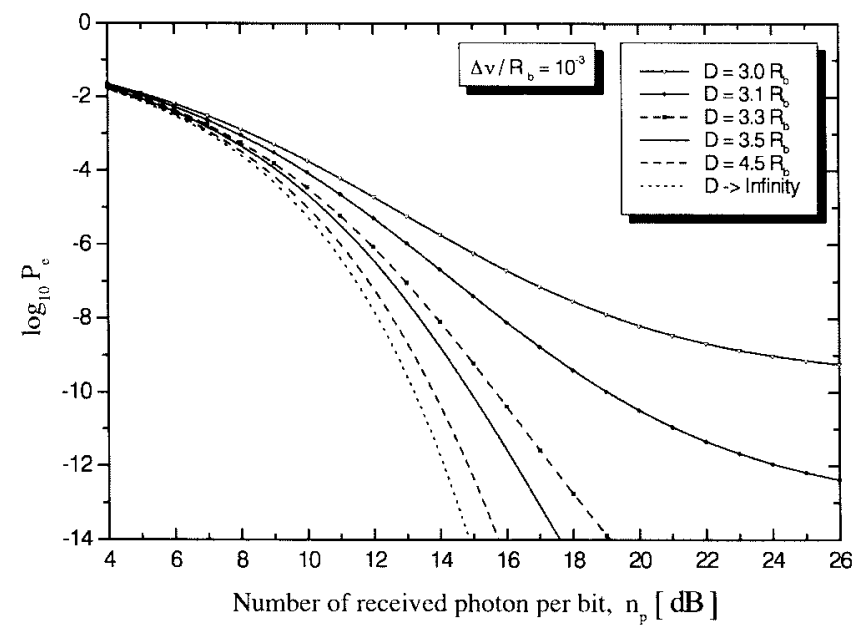

Fig. 2. System error probability versus photon number per bit for linear PLL model.

with variance $1 / \rho$ ) the system error probability dependence on the number of photon per bit is shown in Fig. 2 for the different optical channel spacings.

In the case of the nonlinear second-order PLL model the corresponding dependence, for the fixed bit rate $R_{\mathrm{b}}=1$ $\mathrm{Gb} / \mathrm{s}$, is shown in Fig. 3. The optical channel spacing is used as parameter. The curve with $D=3.1 R_{\mathrm{b}}$ is split into three regions for explanation of the system error probability variations caused by change in the number of photons per bit $n_{\mathrm{p}}$. In the $\mathrm{AB}$ region, the error probability decreases sharply with parameter $n_{\mathrm{p}}$ increasing due to the fact that for moderate values of $n_{\mathbf{p}}$ the changes in parameter $\rho$ are considerable. For example, if the photon number per bit is changed from $12 \mathrm{~dB}$ to $15 \mathrm{~dB}$ the error probability decreases 77.99 times. In the $\mathrm{BC}$ region the effect of the rise of reference-carrier power is compensated by increasing the noise power, so that the system error probability changes are small. For example, if the photon number per bit is changed from $21 \mathrm{~dB}$ to $24 \mathrm{~dB}$ the error probability decreases only 4.69 times. From Fig. 3 it also can be noticed that the saturation effect appears. Namely, as the number of photon per bit $\mathrm{np}$ approaches to infinity the expressions for the optimum loop bandwidth, the equivalent SNR in the loop bandwidth and the SNR at the detector input become, respectively

$$
\begin{aligned}
B_{\text {PLL }, \text { opt }} & =\sqrt{\frac{\pi(r+1) \Delta \nu R_{b} /(2 r)}{\frac{N_{4} R_{b}}{4}+\frac{N_{5} R_{b}}{16}}}, \\
\rho & =1 \sqrt{\frac{2 \pi(r+1)\left(\Delta \nu / R_{b}\right)}{r}\left(\frac{N_{4} R_{b}}{4}+\frac{N_{5} R_{b}}{16}\right)}
\end{aligned}
$$

and

$$
\mathrm{SNR}=2 / \sqrt{\mathrm{N}}
$$

i.e., they are independent on the photon number per bit and the system error probability tends to constant value (BER floor) determined by the laser phase noise (for the fixed optical channel spacing value).

In the case of the linear PLL model, for $D=3.3 R_{\mathrm{b}}$, in order to achieve the error probability of $10^{-9}$ the required photon

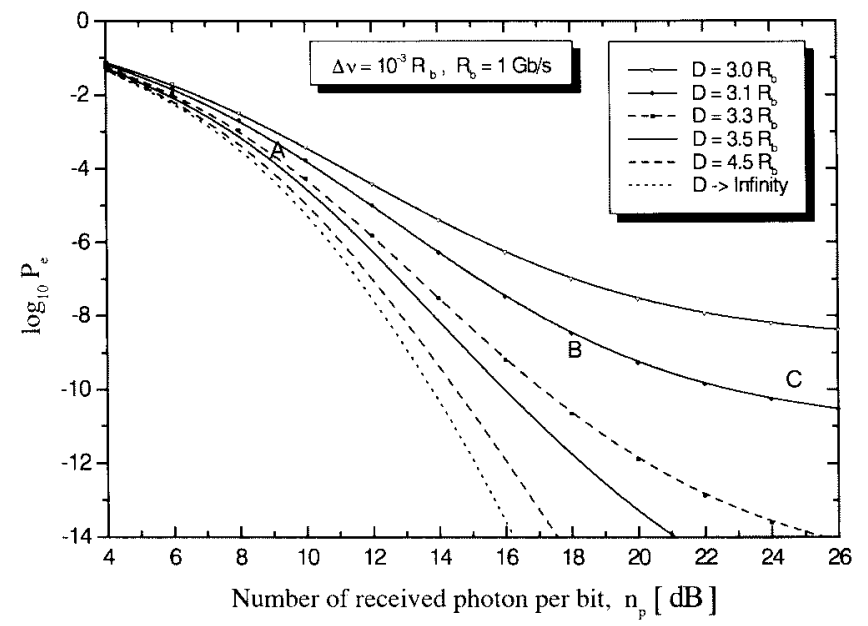

Fig. 3. System error probability versus photon number per bit for nonlinear second-order PLL model.

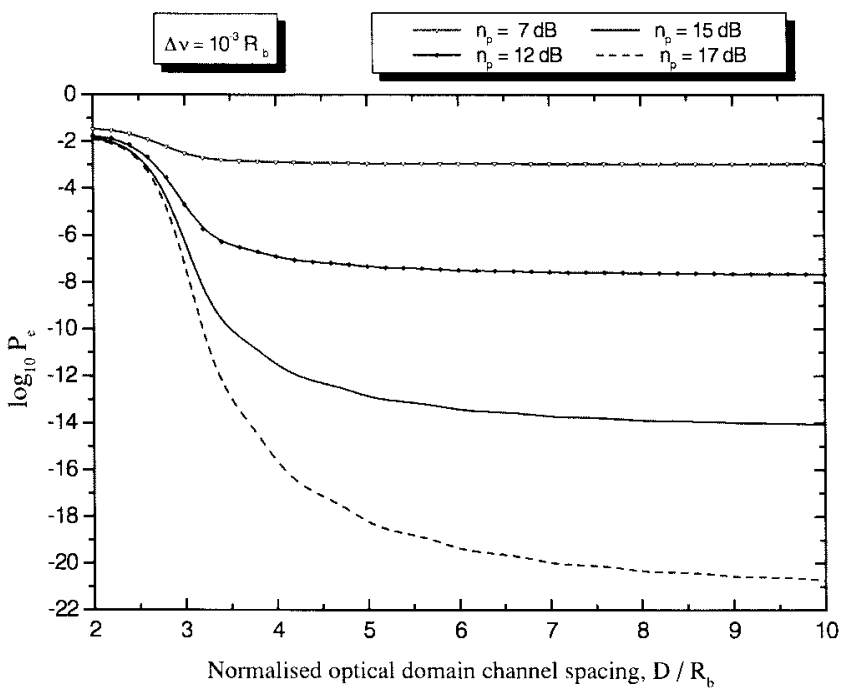

Fig. 4. System error probability versus normalized channel spacing in optical domain for linear PLL model.

number per bit $\left(n_{\mathrm{p}}\right)$ is $14.8 \mathrm{~dB}$, while the error probability $10^{-10}$ can be achieved for $n_{\mathrm{p}}=15.7 \mathrm{~dB}$. In the case of the nonlinear PLL model, for $D=3.3 R_{\mathrm{b}}$, in order to achieve the error probability of $10^{-9}$ the required photon number per bit is $15.8 \mathrm{~dB}$, while the error probability $10^{-10}$ can be achieved for $n_{\mathrm{p}}=17.1 \mathrm{~dB}$. Therefore, the approximation error (with respect to the photon number per bit) when the desired error probability is $10^{-9}$ is not greater than $1 \mathrm{~dB}$, while for the error probability $10^{-10}$ the approximation error is $1.4 \mathrm{~dB}$. Thus, the better the performance is desired the approximation error is greater.

In the case of the linear PLL model the system error probability dependence on the normalized channel spacing in optical domain is shown in Fig. 4. The number of photon per bit is used as parameter.

In the case of the nonlinear second-order PLL model the corresponding dependence, for the fixed bit rate $R_{\mathrm{b}}=1 \mathrm{~Gb} / \mathrm{s}$, is shown in Fig. 5. When $D$ is increased from $3 R_{\mathrm{b}}$ to $5 R_{\mathrm{b}}$ the error probability decreases rapidly. This phenomenon can be 


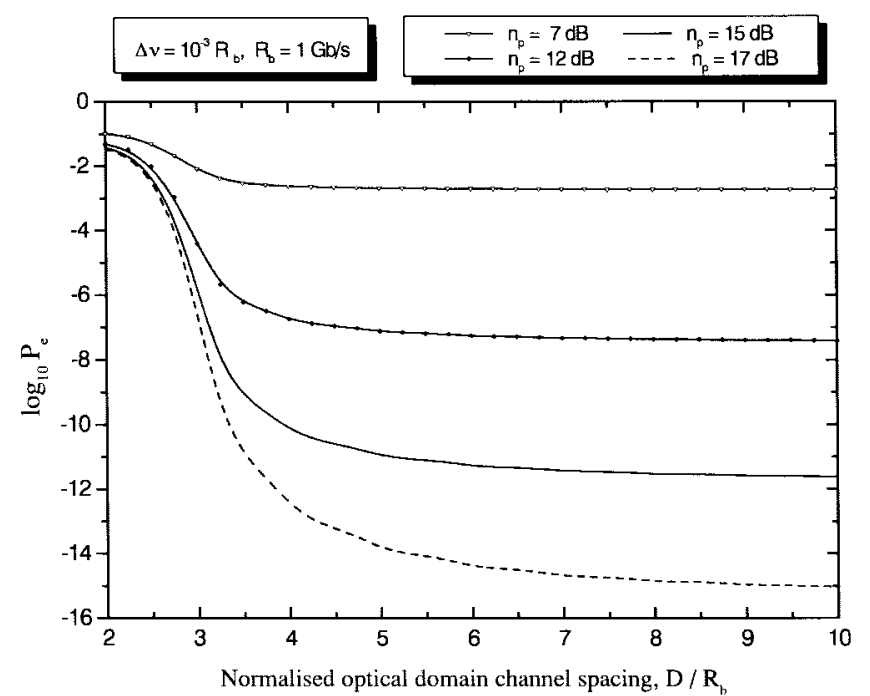

Fig. 5. System error probability versus normalized channel spacing in optical domain for nonlinear PLL model.

explained as follows. For $D<4 R_{\mathrm{b}}$ a part of the power spectral density of the first adjacent channel is within the bandwidth of the bandpass filter. Therefore, as $D$ increases from $3 R_{\mathrm{b}}$ to $4 R_{\mathrm{b}}$ a large amount of the interference power is removed. For $D>4 R_{\mathrm{b}}$ the secondary lobes of the PSD are successfully removed from the IF filter, thus the power removed is less and less important and the error probability decreases more slowly. For example, when $n_{\mathrm{p}}=15 \mathrm{~dB}$, if $D$ is changed from $3 R_{\mathrm{b}}$ to $4 R_{\mathrm{b}}$ the error probability decreases $1.9 \cdot 10^{4}$ times. However, when $D$ is changed from $5 R_{\mathrm{b}}$ to $6 R_{\mathrm{b}}$ the error probability decreases only times times.

In the case of the linear PLL model, for $n_{\mathrm{P}}=15 \mathrm{~dB}$, in order to achieve the error probability of $10^{-9}$ the needed spacing in optical domain is $D=3.3 R_{\mathrm{b}}$, while the error probability $10^{-10}$ can be achieved with $D=3.5 R_{\mathrm{b}}$. In the case of the nonlinear PLL model, for $n_{\mathrm{P}}=15 \mathrm{~dB}$, in order to achieve the error probability $10^{-9}$ the needed spacing in optical domain is $D=3.5 R_{\mathrm{b}}$, while the error probability $10^{-10}$ can be achieved with $D=3.95 R_{\mathrm{b}}$. Thus, in order to achieve the same error probability in the the case of the nonlinear model as that in the case of the linear PLL model, for the same received number of photon per bit, the greater values of the channel spacing in optical domain is required.

\section{CONCLUSION}

A coherent optical PSK receiver with Costas loop in multichannel environment is considered in this paper. The expression for the error probability determination is derived on the basis of the nonlinear second-order PLL model and a Gaussian approximation for filtered adjacent channels. The laser phase noise, the shot noise and the adjacent channel interferences are taken into consideration. The laser phase noise is described through the normalized frequency fluctuations instead of the phase ones. The shot noise of the corresponding photodiodes and adjacent channel interferences are described through the SNR in the loop bandwidth.
The approach and results presented here, can be applied in practice of the heterodyne PLL-based coherent optical receivers design in multichannel environment. For the given channel separation in optical domain $D$ and the laser linewidth $\left(\Delta \nu / R_{b}\right)$, it is possible to calculate the required number of photons per bit in order to achieve the desired error probability. For the same $D$ and $\Delta \nu / R_{b}$ when the nonlinear PLL model is used, the greater value of the number of photons per bit is required in order to achieve the same error probability than that for the linear PLL model.

In designing a system, follow these steps.

1) Choose the value for $r=4 \zeta^{2}, F$ (tipically $r=2$, $F=0.002), R_{\mathrm{b}}$ and $D$.

2) Fix a desired value of the error probability, $P_{\mathrm{e}}$.

3) Fix a desired value of the equivalent loop noise bandwidth, $B_{\text {PLL. }}$.

4) Using expression (18) and model given previously plot the error probability in dependence on the photon number per bit, $n_{p}=R P_{S} /\left(q R_{b}\right)$.

5) Determine the needed photon number per bit, i.e., received optical power in order to achieve the desired error probability.

6) Starting from (18) for the photon number per bit determined in step 5) in calculating $P_{\mathrm{e}}$ determine SNR in loop bandwidth $\rho$.

7) Starting from the following expression

$$
\begin{aligned}
\rho=1 /\left[\frac{\pi\left(1+4 \zeta^{2}\right) \Delta \nu}{8 \zeta^{2} B_{\mathrm{PLL}}}+\left(\frac{q^{2} B}{R^{2} P_{S}^{2}}+\frac{q}{R P_{S}}\right.\right. \\
\left.\left.+\frac{q}{4 R P_{S}} N_{3}+\frac{N_{4}}{4}+\frac{N_{5}}{16}\right) B_{\mathrm{PLL}}\right]
\end{aligned}
$$

determine the laser linewidth.

Therefore, using the previous design procedure, for appropriate chosen parameters $r, F, R_{\mathrm{b}}, D$ and $B_{\text {PLL }}$ it is possible to determine the required received optical power and the laser linewidth.

\section{APPENDIX A \\ The Nonlinear PLL TheOry Basis}

The Costas loop model that has been considered most frequently in literature is shown in Fig. 1(b) (the bottom picture). It has been shown [7]-[9], that we may describe system operation by the stochastic differential equation (see Fig. 1(b), the bottom picture)

$$
\dot{\Phi}(t)=2 \dot{\Psi}(t)-K F(p)\left\lfloor A^{2} \sin \Phi(t)+v(t, \Phi(t))\right\rfloor
$$

where

$$
\begin{aligned}
v(t, \Phi(t))= & \left\lfloor-N_{c}^{2}(t)+N_{s}^{2}(t)-2 A d(t) N_{s}(t)\right\rfloor \sin \Phi(t) \\
& +\left[2 A d(t) N_{c}(t)-2 N_{c}(t) N_{S}(t)\right] \cos \Phi(t) .
\end{aligned}
$$

$\Phi(t)=2 \varphi(t), \varphi(t)=\Psi(t)-\phi_{\mathrm{VCO}}(t)$ represents the actual phase error being tracked by the loop, with being the PLL estimate of $\Psi(t) . F(p)$ is the transfer function of the loop filter in the operator form where $p=(d / d t)$ is the Heaviside operator, $A^{2}$ is the power in the input PLL signal component, $K$ is the open loop gain. $N_{c}(t)$ and $N_{s}(t)$ are respectively the 
in-phase and quadrature components of the narrowband noise at the bandpass filter output. $d(t)$ is -1 or 1 according to the transmitted bit. Using the approximation $\sin 2 \varphi(t) \cong 2 \varphi(t)$ when $\varphi(t)$ is small, a linearized PLL model results. However, this approximation can not be applied always, especially in the case of the practical communications systems analysis. The clearest contributions relative to the statistical dynamics of the phase error process are given in [7] and [9], where the Fokker-Planck equation is solved in a more exact fashion than it had previously been considered.

If we write the loop filter transfer function in the partial fraction expression

$$
F(p)=F_{0}+\sum_{k=1}^{N} \frac{1-F_{k}}{1+\tau_{k} p}
$$

substitute (A2) into (A1) and assume an input of the form $\Psi(t)=\Omega t+\theta$ where $\theta$ is a constant, we can write (A1) as

$$
\begin{aligned}
\dot{\Phi}(t)= & 2 \Omega-F_{0} K\left\lfloor A^{2} \sin \Phi(t)+v(t, \Phi(t))\right\rfloor-\sum_{k=1}^{N} \\
& \cdot \frac{1-F_{k}}{1+\tau_{k} p}\left[A^{2} K \sin \Phi(t)+K v(t, \Phi(t))\right]
\end{aligned}
$$

where $\Omega=\omega_{t r}-\omega_{\text {VCO }}$ is the loop detuning. Introducing the state variable

$$
y_{k}(t)=-K\left[\frac{1-F_{k}}{1+\tau_{k} p}\right]\left[A^{2} \sin \Phi(t)+v(t, \Phi(t))\right]
$$

for $k=1,2, \ldots, N$, we can replace (A3) by the equivalent system of $(N+1)$ first-order stochastic differential equations, i.e.,

$$
\begin{gathered}
\dot{\Phi}(t)=2 \Omega-F_{0} K\left[A^{2} \sin \Phi(t)+v(t, \Phi(t))\right]+\sum_{k=1}^{N} y_{k}(t) \\
\dot{y}_{k}(t)=-\frac{y_{k}(t)}{\tau_{k}}-\frac{\left(1-F_{k}\right) K\left\lfloor A^{2} \sin \Phi(t)+v(t, \Phi(t))\right\rfloor}{\tau_{k}} \\
k=1,2, \cdots, N
\end{gathered}
$$

where $y_{0}=\Phi$. Written this way, it is clear from (A5) that the coordinates $y_{0}, y_{1}, \cdots, y_{N}$ form components of a $(N+1)$ dimensional Markov vector $(\mathrm{y}, t)=\left(\Phi, y_{1}, \cdots, y_{N}, t\right)$ since each component, $\hat{y}_{k}$, depends only upon the present values of $(\mathrm{y}, t)$ and a "white" Gaussian noise process. The most complete characterization of the state $y$ of the tracking loop is its statistical description by means of the transition pdf, viz., $P\left(\mathrm{y}, t \mid \mathrm{y}_{0}, t_{0}\right)$. The transition pdf $P(\mathrm{y}, t)=P\left(\mathrm{y}, t \mid \mathrm{y}_{0}, t_{0}\right)$, of course, can be formally determined by Markov processes theory use.

Given the fact that the components of $\mathrm{y}$ form a vector Markov process. $P(\mathrm{y}, t)$ satisfies the $(N+1)$-dimensional Fokker-Planck (F-P) equation [7]

$$
\begin{array}{r}
\frac{\partial P(\mathrm{y} ; t)}{d t}+\sum_{k=0}^{N} \frac{\partial}{\partial y_{k}} \cdot\left\{\left[K_{k}(\mathrm{y}, t)-\frac{1}{2}\right.\right. \\
\left.\left.\cdot \sum_{l=0}^{N} \frac{\partial}{\partial y_{l}} K_{l k}(\mathrm{y}, t)\right]\right\} P(\mathrm{y} ; t)=0
\end{array}
$$

where the intensity coefficients $K_{k}(\mathrm{y}, t)$ and $K_{l k}(\mathrm{y}, t)$ are defined by the formulas

$$
\begin{aligned}
K_{k}(\mathrm{y}, t) & =\lim _{\Delta t \rightarrow 0} \frac{E\left[\Delta y_{k} \mid \mathrm{y}\right]}{\Delta t}, \text { and } \\
K_{l k}(\mathrm{y}, t) & =\lim _{\Delta t \rightarrow 0} \frac{E\left[\Delta y_{l} \Delta y_{k} \mid \mathrm{y}\right]}{\Delta t}
\end{aligned}
$$

where $E[\cdot \mid \mathrm{y}]$ denotes mathematical expectation of the enclosed quantity given $\mathrm{y}$ and $\Delta y_{k}=y_{k}-\bar{y}_{k}$.

By solving the Fokker-Planck equation setting for the PLL system shown in Fig. 1(b) (the bottom picture) the pdf of the phase error for the $(N+1)$-order PLL is obtained as the result [9]

$$
\begin{aligned}
p(\Phi)= & \frac{\exp [\beta(N) \Phi+\alpha(N) \cos \Phi]}{4 \pi^{2} \exp (-\pi \beta(N))\left|I_{j \beta}(\alpha(N))\right|^{2}} \\
& \cdot \int_{\Phi}^{\Phi+2 \pi} \exp [-\beta(N) x-\alpha(N) \cos x] d x
\end{aligned}
$$

where

$$
\begin{aligned}
\alpha(N)= & \frac{4}{N_{s q} F_{0}^{2} K^{2}}\left[A^{2} K F_{0}-\frac{K N_{s q}}{4 A^{2} \sigma_{G}^{2}} \sum_{k=1}^{N} \frac{1-F_{k}}{\tau_{k}}\right] \\
\beta(N)= & \frac{4}{N_{s q} F_{0}^{2} K^{2}}\left[2 \Omega-A^{2} K \overline{\sin \Phi} \sum_{k=1}^{N}\left(1-F_{k}\right)\right. \\
& \left.\cdot\left(1+\frac{N_{s q}}{4 A^{4} \tau_{k} \sigma_{G}^{2}}\right)\right]
\end{aligned}
$$

where $\Omega=\omega_{I F}-\omega_{\mathrm{VCO}}$ is the frequency offset,

$$
\begin{aligned}
\sigma_{G}^{2} & =\int_{-\pi}^{\pi}(\sin \Phi-\overline{\sin \Phi})^{2} p(\Phi) d \Phi \quad \text { and } \\
N_{s q} & =4 A^{2} N_{0} S_{L}^{-1}
\end{aligned}
$$

with

$$
S_{L}^{-1}=1+\frac{2}{A^{2} N_{0}} \int_{-\infty}^{\infty} R_{N_{c}}^{2} d \tau
$$

being the "squaring lost." $N_{0}$ is the power spectral density of the noise process at the bandpass filter input.

In the case of the first-order PLL, when the loop filter is omitted, $(N=0, F(p)=1)$ the previous equations reduce to

$$
\begin{aligned}
\alpha & =\frac{4 A^{2}}{N_{s q} K} \\
\beta & =\frac{4}{N_{s q} K^{2}} 2 \Omega=\beta_{0} \Omega
\end{aligned}
$$

what is consistent with expressions (26) and (27) in [12] that refer to standard PLL. If the signal amplitude $A$ is constant then we can write $\alpha=\alpha_{0}$. In practice the PLL receiver cannot be realized without corresponding loop filter, and therefore we are obliged to use the second-order PLL model.

In the case of the second-order PLL $\left(N=1, F_{0}=F_{1}=\right.$ $\left.F=\tau_{2} / \tau_{1}, F(p)=\left(1+\tau_{2} p\right) /\left(1+\tau_{1} p\right)\right)$ (A9) reduces to

$$
\begin{aligned}
& \alpha=\frac{r+1}{r} \rho^{\prime}-\frac{1-F}{r \sigma_{G}^{2}} \\
& \beta=\frac{r+1}{r}\left[\frac{2 \Omega}{A^{2} K}-\overline{\sin \Phi}\right]+\alpha \overline{\sin \Phi}
\end{aligned}
$$


where $r=A^{2} K F \tau_{2}$ is the loop damping parameter $(\zeta=$ $\sqrt{r / 4}$-the loop damping factor) and $W_{L}=(r+1) /\left(2 \tau_{2}\right)$ (for $r \tau_{1} \gg \tau_{2}$ ) is the loop bandwidth as defined from linear tracking theory, i.e.

$$
W_{L}=2 B_{\mathrm{PLL}}=\frac{1}{2 \pi j} \int_{-j \infty}^{j \infty}\left|H_{\mathrm{PLL}}(s)\right|^{2} d s
$$

with $H_{\text {PLL }}(s)$ being the closed transfer function when the loop is linearized [7]-[8]. $\rho^{\prime}=(\rho / 4) S_{L}$ is the effective SNR in the loop bandwidth, while $\rho=2 A^{2} / N_{0} W_{L}$ is the SNR in the loop bandwidth. Therefore, while determining the SNR in the loop bandwidth the linear PLL theory is to be used.

The validity of using (A8) to represent the statistical properties of the phase error in a second-order loop, in the nonlinear region, was verified by comparing the equation graphically with an experimentally derived pdf in $[8$, p. 33].

In order to make the comparison between linear and nonlinear theory the phase error variance versus the SNR in the loop was shown in Fig. 7 of the paper [7]. For $\rho>4$ the approximation of the nonlinear PLL model by the linear one is justified.

The results of reference [7]-[9] are applicable for phase error only due to additive noise (i.e. shot noise in the case of optical communications). In that case the normalized frequency offset

$$
\Omega_{n}=\Omega / A^{2} K=\left(\omega_{I F}-\omega_{\mathrm{VCO}}\right) / A^{2} K
$$

is constant. This situation is applicable in the case of classical digital communications where the phase noise of oscillators can be neglected. In the case of optical communications the phase noise of laser oscillators cannot be neglected and the normalized frequency offset becomes stochastic variable

$$
\Omega_{n}=\dot{\varphi} / A^{2} K
$$

where $\varphi(t)$ represents the actual phase error being tracked by the loop $(\dot{\varphi}=d \varphi(t) / d t)$, and the method of conditional probabilities [8] is to be used. That is, we shall develop expressions for the particular parameter, conditioned on the normalized frequency offset. By averaging over this condition, which is random, we determine the behavior of interest. Therefore, the conditional pdf of phase error due to the phase noise and shot noise, as well as adjacent channel interferences, in the case of the second-order PLL, according to (A8) becomes

$$
\begin{aligned}
p\left(\Phi \mid \Omega_{n}\right)= & \frac{\exp \left[\alpha \cos \Phi+\beta\left(\Omega_{n}\right) \Phi\right]}{4 \pi^{2} \exp \left[-\pi \beta\left(\Omega_{n}\right)\right]\left|I_{j \beta\left(\Omega_{n}\right)}(\alpha)\right|^{2}} \\
& \cdot \int_{\Phi}^{\Phi+2 \pi} \exp \left[-\alpha \cos x-\beta\left(\Omega_{n}\right) x\right] d x
\end{aligned}
$$

where [see (A11)]

$$
\begin{aligned}
\alpha & =\frac{r+1}{r} \rho^{\prime}-\frac{1-F}{r \sigma_{G}^{2}} \\
\beta\left(\Omega_{n}\right) & =\frac{r+1}{r} \frac{\rho^{\prime}}{F}\left[2 \Omega_{n}-\overline{\sin \Phi}\right]+\alpha \overline{\sin \Phi .}
\end{aligned}
$$

So, the shot noise of the corresponding photodiodes and adjacent channel interferences are taken into consideration through the SNR in the loop bandwidth, whereas the laser phase noise is described through the normalized frequency offset.

\section{APPENDIX B CONDITIONAL RECEIVER ERROR PROBABILITY DETERMINATION}

The data detector considered here contains the low-pass filter, the sampler and the estimator. The signal $v_{I}(t)$ is filtered by a low-pass filter, represented by an integrate and dump filter with an integration constant equal the bit duration $T$. The sampler output at time $k T$, according to [3], is

$$
\begin{aligned}
z(k T)= & \frac{A_{\mathrm{VCO}} d_{n}(k T) r_{L} R \sqrt{P_{S} P_{L O}}}{T} \int_{0}^{T} \cos \varphi(t) d t \\
& +\frac{1}{T} \int_{0}^{T}\left[n_{I}(t)+c_{I}(t)\right] d t .
\end{aligned}
$$

Since $c_{I}(t)$ and $n_{I}(t)$ are zero-mean Gaussian noises, the total variance can be found as

$$
\begin{aligned}
\sigma_{n_{I}}^{2}= & \sigma_{n}^{2}+\sigma_{c}^{2}=\int_{-B}^{B} \operatorname{PSD}_{n_{I}}(f)\left|H_{i}(f)\right|^{2} d f \\
& +\int_{-B}^{B} \operatorname{PSD}_{c_{I}}(f)\left|H_{i}(f)\right| d f
\end{aligned}
$$

where $H_{i}(f)$ is the transfer function of the integrate and dump filter [3]

$$
H_{i}(f)=\frac{\sin \pi f T}{\pi f T}
$$

Knowing that

$$
\begin{aligned}
\operatorname{PSD}_{n_{I}}(f) & =\frac{1}{2} A_{\mathrm{VCO}}^{2} q r_{L}^{2} R P_{L O} \quad \text { and } \\
\operatorname{PSD}_{c_{I}}(f) & =\frac{A_{\mathrm{VCO}}^{2} r_{L}^{2} R^{2} P_{S} P_{L O}}{8} \operatorname{PSD}_{T}(f)
\end{aligned}
$$

the following is valid:

$$
\begin{aligned}
\sigma_{n}^{2} & =\frac{A_{\mathrm{VCO}}^{2} q r_{L}^{2} R P_{L O}}{2} \int_{-B}^{B}\left|H_{i}(f)\right|^{2} d f \\
& \approx \frac{1}{2} A_{\mathrm{VCO}}^{2} q r^{2} R P_{L O} R_{b} \\
\sigma_{c}^{2} & =\frac{A_{\mathrm{VCO}}^{2} r_{L}^{2} R^{2} P_{S} P_{L O}}{8} \int_{-B}^{B} \operatorname{PSD}_{T}(f)\left|H_{i}(f)\right|^{2} d f \\
& =\frac{1}{8} A_{\mathrm{VCO}}^{2} r_{L}^{2} R^{2} P_{S} P_{L O} \mathrm{~N} .
\end{aligned}
$$

where $\mathrm{N}$ is defined by (17).

It is well known [5]-[10] that for moderate values of $B_{\text {PLL }}$ $\left(B_{\text {PLL }}<0.25 R_{b}\right) \varphi(t)$ can be taken to be constant during the bit period, thus

$$
z(k T) \cong A_{\mathrm{VCO}} d_{n}(k T) r_{L} R \sqrt{P_{S} P_{L O}} \cos \varphi+n_{l}(k T)
$$

and the conditional error probability to be used

$$
P_{e}(\varphi)=\frac{1}{2} \operatorname{erfc}[\operatorname{SNR} \cos \varphi]
$$


where

$$
\mathrm{SNR}=\frac{1}{\sqrt{2}} \frac{A_{\mathrm{VCO}} r_{L} r_{L} R \sqrt{P_{S} P_{L O}}}{\sqrt{\sigma_{n}^{2}+\sigma_{c}^{2}}}=\frac{1}{\sqrt{\frac{\mathrm{N}}{4}+\frac{1}{n_{p}}}}
$$

is the SNR at the data detector input with $n_{P}=\left(R P_{S} / q R_{b}\right)$ being the number of photon per bit. This expression is different from expression (39) in the paper [3]. Namely, the expression (36) in [3] was wrongly determined and results given in [3] cannot be repeated using expression (39). If $N$ is the number of adjacent channels then procedure for the receiver error probability calculation, given in [3], fails.

Finally, the expression for the receiver error probability can be calculated by averaging the conditional error probability per $\varphi$. In the case of the linear PLL model the distribution of the stochastic process $\varphi$ a zero-mean Gaussian, while that in the case of the nonlinear PLL model is described in Appendix A.

Note that for a single-channel system $(N=0)$ SNR becomes

$$
\mathrm{SNR}=\sqrt{n_{P}}=\sqrt{\frac{R P_{S}}{q R_{b}}}
$$

what is consistent with previously reported results that refer to a single-channel system [1].

\section{REFERENCES}

[1] L. Kazovsky, S. Benedetto, and A. E. Willner, Optical Fiber Communication Systems, 1996.

[2] L. G. Kazovsky, "Sensitivity penalty in multichannel coherent optical communications," J. Lightwave Technol., vol. 6, pp. 1353-1365, 1987.

[3] D. Zaccarin, D. Angers, and T. H. Huynh, "Performance analysis of optical heterodyne PSK receivers in the presence of phase noise and adjacent channel interference," J. Lightwave Technol., vol. 8, pp. 353-366, Mar. 1990.

[4] S. P. Majumder, M. S. Alam, and R. Gangopadhyay, "Effect of nonuniform FM response on the performance of multichannel heterodyne FSK systems using optical amplifiers," J. Lightwave Technol., vol. 15, pp. 188-193, 1997.

[5] M. C. Stefanovic, I. B. Djordjevic, G. T. Djordjevic, and J. V. Basta, "Coherent optical heterodyne PSK receiver in multichannel environment," J. Optic. Commun., vol. 20, no. 1, pp. 12-15, Feb. 1999.

[6] M. C. Stefanovic, G. T. Djordjevic, and I. B. Djordjevic, "Performance of binary CPSK satellite communication system in the presence of noises and noisy carrier reference signal," in Proc. Int. J. Electron. Commun. (AEU), vol. 53, no. 2, pp. 70-76, 1999.

[7] W. C. Lindsey, "Nonlinear analysis of generalized tracking systems," Proc. IEEE, vol. 57, pp. 1705-1722, Oct. 1969.

[8] W. C. Lindsey and M. K. Simon, Telecommunication Systems Engineering. Englewood Cliffs, NJ: Prentice-Hall, 1973.

[9] W. C. Lindsey and M. K. Simon, "The performance of suppressed carrier tracking loops in the presence of frequency detuning," Proc. IEEE, vol. 58, pp. 1315-1321, Sept. 1970.

[10] I. B. Djordjevic, "Performance analysis and optimization of coherent optical systems with phase-locked loop," Ph.D. dissertation, Univ. Nis, Yugoslavia, Jan. 1999.

[11] A. Blanchard, Phase-Locked Loops: Application to Coherent Receiver Design. New York: Wiley, 1976.

[12] A. J. Viterbi, "Phase-locked loop dynamics in the presence of noise by Fokker-Planck techniques," Proc. IEEE, vol. 51, pp. 1737-1753, 1963.

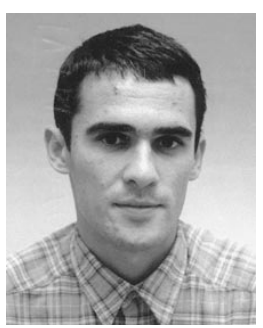

Ivan B. Djordjevic was born in Nis, Yugoslavia, in 1969. He received the B.S., M.S., and Ph.D. degrees in electrical engineering from the Faculty of Electronic Engineering, University of Nis, in 1994 1997, and 1999, respectively.

His research interests are coherent optical systems with PLL, synchronization, communication theory, and coding theory.

Mihajlo C. Stefanovic (A'92) was born in Nis, Yugoslavia, in 1947. He received the B.S., M.S., and Ph.D. degrees in electrical engineering from the Faculty of Electronic Engineering (Department of Telecommunications) from the University of Nis.

$\mathrm{He}$ is currently a Professor with the Faculty of Electronic Engineering, University of Nis. His primary research involves statistical communication theory, optical communications, and satellite communications. His areas of interests also include applied probability theory, optimal receiver design, and synchronization. 